\title{
MR-based attenuation correction for PET/MRI neurological studies with continuous-valued attenuation coefficients for bone through a conversion from $\mathrm{R2}^{*}$ to $\mathrm{CT}$-Hounsfield units
}

\author{
Meher R Juttukonda ${ }^{1,2}$, Bryant G Mersereau ${ }^{1,2}$, Yasheng Chen ${ }^{2,3}$, Yi Su$^{4}$, Brian G Rubin ${ }^{4}$, \\ Tammie LS Benzinger ${ }^{4,5}$, David S Lalush ${ }^{1,2}$, and Hongyu An ${ }^{1,3}$ \\ ${ }^{1}$ Joint Department of Biomedical Engineering, University of North Carolina - Chapel Hill, Chapel \\ Hill, NC 27599 \& North Carolina State University, Raleigh, NC 27695 \\ 2Biomedical Research Imaging Center, University of North Carolina Chapel Hill, Chapel Hill, NC \\ 27599 \\ ${ }^{3}$ Department of Radiology, University of North Carolina Chapel Hill, Chapel Hill, NC 27599 \\ ${ }^{4}$ Mallinckrodt Institute of Radiology, Washington University, St. Louis, MO 63130 \\ ${ }^{5}$ Department of Neurological Surgery, Washington University, St. Louis, MO 63130
}

\section{Introduction}

\begin{abstract}
Advances in simultaneous positron emission tomography/magnetic resonance imaging (PET/MRI) scanners have opened up a multitude of opportunities in neurological imaging, including applications in multimodal studies of perfusion, metabolism, receptor status, and function [1-3,5]. PET/MRI systems have numerous advantages over PET/computed tomography (CT) systems such as superior soft tissue contrast, reduced exposure to ionizing radiation, the possibility of MR-based motion correction, and simultaneous acquisition of complementary PET and MR data [27]. However, MR-based correction for photon attenuation in the head remains challenging, particularly for neurological applications requiring quantitation of data [6].
\end{abstract}

PET is an inherently quantitative imaging modality through which estimates of the uptake of a tracer compound can be computed. However, the attenuation of gamma photons, which are the source of PET signal, by body tissues reduces the accuracy of quantitative measurements. The likelihood of photon attenuation in a tissue is governed by both the linear attenuation coefficient (LAC) and the thickness of the tissue. With knowledge of the distribution of tissues present in the PET field-of-view and their respective LACs, it is

\footnotetext{
(C) 2015 Published by Elsevier Inc.

Corresponding Author: Hongyu An, D.Sc., Department of Radiology, CB\# 7513, Chapel Hill, NC - 27599, Phone: (919) 843-8256; Fax: (919) 843-4456; hongyu_an@med.unc.edu.

Publisher's Disclaimer: This is a PDF file of an unedited manuscript that has been accepted for publication. As a service to our customers we are providing this early version of the manuscript. The manuscript will undergo copyediting, typesetting, and review of the resulting proof before it is published in its final citable form. Please note that during the production process errors may be discovered which could affect the content, and all legal disclaimers that apply to the journal pertain.
} 
possible to perform a correction for photon attenuation. This distribution, referred to as an attenuation map or $\mu$-map, is employed as the basis for attenuation correction (AC) during the PET reconstruction process [6].

In PET/CT systems, attenuation maps are obtained through piecewise linear scaling of the CT data in each imaging voxel [7-9]. Such a scaling is possible because CT inherently measures photon attenuation, albeit at a lower energy than used in PET imaging. An analogous conversion from MR images to PET LACs is not possible due to the differences between the proton density- and tissue relaxation-dependent MR signal and the electron density-dependent attenuation process [6]. Bone is particularly affected by these differences since it exhibits near-zero signal in conventional T1-weighted MR (T1-MR) images but is the greatest biological attenuator of photons (per unit volume) in the body. Proper delineation of bone is of particular importance in neurological PET imaging due to the relatively high prevalence of bone found in the head [4]. Previous studies [10,11] have shown that improperly accounting for bone can result in large underestimations of PET signal, particularly in tissue adjacent to bone. As a result, two of the major challenges for MR-based attenuation correction (MRAC) in the head are proper identification of bone and accurate estimation of bone LACs [6].

There are two main classes of MR-based methods for PET/MRI attenuation correction. The first class consists of atlas-based methods [12-15,28,29]. These methods typically rely on a precompiled atlas of paired MR and CT images and an algorithm to generate an artificial CT image (pseudo-CT) from patient MR images. These pseudo-CTs are subsequently converted to PET attenuation maps through the same scaling operation used in PET/CT attenuation correction. The availability of bone information from the CT component of the atlas helps circumvent the previously mentioned problems of bone identification and LAC estimation. Atlas-based methods typically produce relatively accurate PET reconstructions compared to reconstructions performed with CT-based attenuation correction [6]. However, these approaches are computationally intensive, and their accuracy depends on the population anatomical variation represented by the atlas [5].

The second class of MRAC methods consists of segmentation-based methods [16-20]. These approaches differ from their atlas-based counterparts in that they generate $\mu$-maps from patient MR images alone [4]. They function by segmenting patient MR images into tissue classes and then assigning a constant LAC value to all voxels of each tissue class [6]. Methods using Dixon-based fat/water separation were the first to be presented [16,17], but the lack of bone delineation adversely affects the accuracy of these methods in the head. To overcome this problem, a few MRAC methods [18-19] based on ultrashort echo-time (UTE) sequences have been presented. These dual-echo UTE (DUTE) methods aim to identify regions of bone by examining differences in images acquired with and without bone signal present (first and second echo, respectively). Keereman et al. [18] used an approach based on R2* signal decay between the first and second echoes to identify regions of bone and a region-growing approach to identify regions of air. Catana et al. [19] used arithmetic operations on DUTE images after normalization to identify regions of bone and air. Berker et al. [20] presented a method that distinguishes bone/air regions using arithmetic operations on UTE images and differentiates fat/water regions using a Dixon-based separation. Two 
advantages of segmentation-based methods are shorter computation time and better accounting of anatomical variation. However, segmentation-based methods typically produce less accurate PET reconstructions compared to atlas-based methods [4]. This reduced accuracy may result from the inability of most MR sequences to capture bone signal, incorrect segmentation of tissues, and/or the homogeneous representation of bone LACs. Therefore, the goal of this study was to develop an MR-based attenuation correction method for PET/MRI neurological studies that more accurately identifies bone, soft tissue, and air and provides continuous-valued attenuation coefficients for bone.

\section{Methods}

\subsection{Image Acquisition and Pre-processing}

PET/MRI and CT datasets were obtained from 98 subjects (mean age [ \pm standard deviation]: 66 years [ \pm 9.8$], 57$ females, 3 with very mild dementia) at Washington University in St. Louis, MO using an IRB-approved protocol and with informed consent. No participants had comorbidities that could interfere with testing, and participants did not receive additional radiotracer administrations within 24 hours. The enrollment exclusion criteria included contraindications to PET, PET/CT, or PET/MRI (e.g. electronic medical devices, inability to lie still for long periods), known claustrophobia, pregnancy, and breast-feeding.

${ }^{18}$ F-Florbetapir (Amyvid [Avid], Eli Lilly, Indianapolis, IN) PET images were acquired for each subject on a hybrid PET/MRI system (Biograph mMR, Siemens AG, Erlangen, Germany). Subjects were injected with $352 \pm 29 \mathrm{MBq}$ of ${ }^{18} \mathrm{~F}$-Florbetapir tracer, and PET acquisitions were begun either immediately or 50 minutes after injection. CT images of the head were acquired separately using a PET/CT system (Biograph 40 PET/CT, Siemens AG, Erlangen, Germany). Images were acquired at $120 \mathrm{kVp}$ with a voxel size of $0.59 \times 0.59 \times 3.0$ $\mathrm{mm}^{3}$ and a matrix size of $512 \times 512 \times 74$. The CT and PET/MRI images were acquired within $8.3 \pm 6$ days of each other with no surgical procedures in between. All images were deidentified before being transferred off-line for image analysis.

DUTE images were acquired using the VB18 version of the UTE AC sequence provided by the vendor. This sequence is considered a "work-in-progress" (WIP). The following imaging parameters were used: repetition time $(\mathrm{TR}) /$ echo time $1\left(\mathrm{TE}_{1}\right) /$ echo time $2\left(\mathrm{TE}_{2}\right)=$ 2300/0.07/2.46 ms, acquisition time $=1 \mathrm{~min} 40 \mathrm{sec}$, flip angle $=10^{\circ}$, field-of-view $(\mathrm{FOV})=$ $300 \mathrm{~mm}^{2}$, and voxel size $=1.56 \times 1.56 \times 1.56 \mathrm{~mm}^{3}$. Two-point Dixon images were acquired using the vendor-provided Dixon-VIBE AC sequence with the following imaging parameters: $\mathrm{TR} / \mathrm{TE}_{1} / \mathrm{TE}_{2}=2300 / 1.23 / 2.46 \mathrm{~ms}$, acquisition time $=18 \mathrm{sec}$, flip angle $=10^{\circ}$, and voxel size $=2.6 \times 2.6 \times 3.12 \mathrm{~mm}^{3} . \mathrm{T} 1$-weighted $\mathrm{MR}(\mathrm{T} 1-\mathrm{MR})$ images were acquired using a 3-dimensional magnetization-prepared rapid gradientecho (MPRAGE) sequence with the following imaging parameters: $\mathrm{TR} / \mathrm{TE}=2300 / 2.95 \mathrm{~ms}$, inversion time $=900 \mathrm{~ms}$, acquisition time $=5 \mathrm{~min} 11 \mathrm{sec}$, flip angle $=9^{\circ}$, number of partitions $=176, \mathrm{FOV}=256 \mathrm{~mm}^{2}$, and voxel size $=1 \times 1 \times 1.2 \mathrm{~mm}^{3}$.

Prior to processing, the CT and MR images of each subject were first transformed into $\mu$ map space as required by the vendor-provided PET reconstruction program (e7tools, Siemens Medical Solutions, Knoxville, TN). To achieve this transformation, the CT, T1- 
MR, and DUTE images of each subject were registered to the corresponding inphase Dixon image (already in $\mu$-map space) using a 6-parameter rigid model and mutual information as implemented by the FSL Toolbox (FMRIB, Oxford, United Kingdom). Registration was performed instead of resampling because, in addition to performing resampling, registration also accounts for any shift in subject position between scans. The results of these registrations were manually inspected to ensure good alignment. Images were also inspected for artifacts, and it was found that 89 of 98 subjects exhibited some level of dental artifacts. These artifacts were left uncorrected in order to derive attenuation maps as they are often produced in a clinical setting. All intermediate images derived from the MR data were first computed in their native space and subsequently transformed to $\mu$-map space.

\subsection{Tissue Segmentation}

Accurate identification of bone is of paramount importance for two key reasons:

1) it is prominently present in the head, and 2) it has higher LACs than other tissues. In addition, there are three other regions of interest in the head that must be properly identified: air, fat, and soft tissue. R2*, Dixon-Fat, Dixon-Water, and iUTE images were used as intermediate images to segment bone, fat, soft tissue, and air, respectively, using simple thresholding. Figure 1 shows the T1 (Figure 1A), CT (Figure 1B), and the four intermediate images (Figure $1 \mathrm{C}-\mathrm{F}$ ).

2.2.1 Air-Since regions of air exhibit the lowest signal intensities in the UTE echo 1 $\left(\mathrm{UTE}_{1}\right)$ image, the intermediate image for air (Figure 1F) was acquired by computing the voxel-wise multiplicative inverse of the $\mathrm{UTE}_{1}$ image (iUTE). The iUTE images were then normalized to their $99^{\text {th }}$ percentile value before a simple threshold (cutoff $=0.06$ ) was applied to segment air.

The cutoff threshold for air in iUTE images was determined using histogram analysis to preferentially select air voxels over bone and CSF voxels and was identical for all subjects. The UTE sequence parameters produce sufficiently high contrast between air and the rest of the head in the iUTE images, as shown in Figure 1F, to provide consistently good air segmentation across subjects.

2.2.2 Bone-Regions of bone exhibit faster transverse decay characteristics compared to soft tissue. Therefore, the R2* map for each subject was computed in a similar manner as in [18] via Equation 1. These R2* maps were used as the intermediate images for bone segmentation (Figure 1C). $\mathrm{UTE}_{1} / \mathrm{TE}_{1}$ and $\mathrm{UTE}_{2} / \mathrm{TE}_{2}$ represent the images/echo times from the first and second echoes of the DUTE sequence, respectively.

$$
R 2^{*}=\frac{\ln \left(3 \cdot U T E_{1}\right)-\ln \left(U T E_{2}\right)}{T E_{2}-T E_{1}}
$$

Unusually low voxel intensities in the $\mathrm{UTE}_{1}$ image, presumably due to eddy current effects, resulted in negative $\mathrm{R} 2 *$ values in some brain regions. To perform a correction for this phenomenon in post-processing, the $\mathrm{UTE}_{1}$ image of each subject was scaled by an empirically-determined factor of three during R2* computation (Equation 1). This was the 
smallest factor that ensured most brain voxels displayed a higher signal intensity in the $\mathrm{UTE}_{1}$ image than in the $\mathrm{UTE}_{2}$ image, resulting in positive R2* values. This scaling was performed only for the computation of the R2* maps, and the mention of $\mathrm{UTE}_{1}$ elsewhere in this work refers to the original acquired images. Due to the noisy nature of the R2* images, voxels classified as air in Section 2.2.1 were removed from the R2* image prior to thresholding for bone. A simple threshold ( cutoff $=550 \mathrm{~s}^{-1}$ ) was then applied to the R2* maps to identify regions of bone. This threshold value, which is slightly higher than the threshold used by Keereman et al. [18], was chosen to select as many bone voxels as possible while minimizing the number of fat and CSF voxels included. As with the threshold for air, the cutoff threshold for bone segmentation was identical for all subjects in the study.

2.2.4 Fat \& Soft Tissue-The fat and water images computed by the vendor-provided Dixon sequence were used as the intermediate images for fat (Figure 1D) and soft tissue (Figure 1E) segmentation, respectively. Simple thresholds were used to segment both fat (cutoff $=250$ ) and soft tissue (cutoff $=150$ ). A head mask was derived through binary and morphological operations on the $\mathrm{UTE}_{1}$ image, and any voxel in the mask not already classified as air, bone, fat, or soft tissue was given a classification of soft tissue. If a voxel was segmented as both fat and soft tissue, the fat tissue class was given higher priority. This classification of fat and soft tissue also serves to reduce erroneous classifications in noisy regions of R2* images. Thus, the proposed segmentation method is hereby referred to as $\underline{\mathrm{R}} 2 *$ and iUTE-based segmentation with Dixon-based Refinement (RiDR).

\subsection{Mapping CT-Hounsfield units using MR R2*}

As previously noted (Section 1), MR signal intensities have no direct correlation with LAC values. However, certain MR decay parameters may be associated with attenuation coefficients. The parameter R2* represents the rate constant of MR signal decay following $\mathrm{RF}$ excitation. R2* incorporates both the transverse irreversible relaxation rate $\mathrm{R} 2$ as well as the reversible relaxation rate $\mathrm{R} 2$ '. Recent studies investigating the microstructure of bone have shed light on the $\mathrm{R} 2$ characteristics of the different water domains present in bone. According to Horch et al. [24], water present in porous domains (lower density) of cortical bone exhibits a lower R2 value than water present in collagen-bound domains (higher density). Since R2 is a component of R2*, we postulate that R2* values should provide a reasonable measure of bone density, and thus, LAC values.

Regression analysis was performed between R2* and CT-Hounsfield unit (CT-HU) values for each subject using data from voxels classified as bone by both modalities. For CT, any voxel with a value greater than $100 \mathrm{HU}$ was classified as bone [23]. This relatively low CT threshold was chosen to provide a complete characterization of the relationship between $\mathrm{R} 2 *$ and $\mathrm{CT}$ in bone. For R2*, any voxel with a value greater than $500 \mathrm{~s}^{-1}$ was classified as bone [18], which is slightly lower than the R2* threshold used for the segmentation in Section 2.2.2. This lower value was chosen to include as many bone voxels as possible when deriving a relationship between MR R2* and CT-HU with the knowledge that misclassifications will be minimized after intersection with the CT classification, a luxury not available during segmentation. 
Due to high levels of noise present in the $\mathrm{R} 2 *$ maps, a voxel-by-voxel comparison between R2* and CT-HU was avoided. Instead, the following spatially-mapped binning approach was followed. For each subject, the R2* values of bone voxels were sorted numerically and divided into 100 bins, each containing an equal number of voxels. For all voxels within an $\mathrm{R} 2 *$ bin, the CT-HU values were matched through spatial correspondence from the aligned CT image. The mean R2* and CT-HU values of each bin were then computed and plotted for the first 98 bins. The last two bins were excluded due to high levels of noise. A fiveparameter sigmoid model (Equation 2) was fit to the mean data using the following parameters and conventions: $\mathrm{A}=$ lower horizontal asymptote, $\mathrm{B}=$ steepness (positive), $\mathrm{C}=$ inflection point, $\mathrm{D}=$ higher horizontal asymptote, and $\mathrm{G}=$ asymmetry of steepness [30].

$$
C T=\mathrm{D}+\frac{A-D}{\left[1+\left(\frac{\mathrm{R} 2^{*}}{\mathrm{C}}\right)^{\mathrm{B}}\right]^{\mathrm{G}}}
$$

In order to validate the regression model, a "leave-one-out" procedure was employed. For each subject, an R2* vs. CT-HU curve was generated from the remaining 97 subjects using the procedure described in the preceding paragraph. The sigmoid model was derived for a given subject by fitting data (MATLAB and "Five Parameter Logistic Regression", MATLAB Central File Exchange, The Mathworks, Inc., Natick, MA) from the remaining 97 subjects, which produced a conversion equation to estimate CT values from R2*. A sigmoid relationship derived for an example subject (Figure 2) indicates a strong $\left(\mathrm{r}^{2}=0.95\right)$ correlation between mean R2* and CT-HU values. This conversion, dubbed the continuousvalued attenuation coefficients from $\underline{\mathrm{R}} 2 *$ (CAR) method, was then employed to generate the bone portion of the PET attenuation maps (Section 2.4).

\subsection{Formation of Attenuation Maps}

Two attenuation maps were generated for each subject: the gold standard CT-based map and the map derived from the proposed CAR-RiDR method. The vendor-provided Dixon-based attenuation map was not included due to known poor performance resulting from ignoring bone $[4,6,25]$.

As presented by Carney et al. [8], the gold standard CT-based map $\left(\mu_{\mathrm{CT}}\right)$ was derived for each subject through piecewise linear scaling of the subject CT image. As shown in Equation 3, voxels with values $<50 \mathrm{HU}$ (air-tissue mix) were scaled differently than voxels with values $\geq 50 \mathrm{HU}$ (tissue-bone mix) [8].

$$
\begin{array}{rlrll}
\text { Below } & 50 & H U: \mu & =9.6 \times 10^{-5} \cdot(H U+1000) \quad \mathrm{cm}^{-1}, \\
\text { Above } & 50 & H U: \mu & =5.1 \times 10^{-5} \cdot(H U+1000)+4.71 \times 10^{-2} & \mathrm{~cm}^{-1},
\end{array}
$$

The RiDR segmentation for bone, air, fat, and soft tissue was used as the basis for the proposed attenuation map ( $\left.\mu_{\mathrm{CAR}-\mathrm{RiDR}}\right)$. First, each tissue class (excluding bone) was assigned a constant PET LAC value: air $=0 \mathrm{~cm}^{-1}$ [19], fat $=0.092 \mathrm{~cm}^{-1}$ [26], and soft tissue $=0.1 \mathrm{~cm}^{-1}[16]$. Next, R2* values in voxels classified as bone were converted to CT-HU values using the CAR relationship described in Section 3.2. These estimated CT values were 
translated to PET attenuation coefficients using the same piecewise linear scaling [8] employed by the gold standard method.

\subsection{PET Data Reconstruction}

PET data for all subjects were acquired from both the head and neck regions. However, acquired $\mathrm{CT}$ images had inadequate coverage of the neck region, resulting in gold standard attenuation maps that did not capture the PET FOV. This discrepancy in imaging coverage was addressed by replacing affected slices in the gold standard map with slices from the vendor-provided MR Dixon-based attenuation map, which provided full coverage of the head and neck. To ensure fair comparison, this replacement was also performed in the $\mu_{\text {CAR-RiDR map. }}$

The e7Tools program was used to reconstruct raw list-mode PET data. For each subject, two reconstructions were performed, one using each attenuation map. PET images were reconstructed using an ordered subset expectations maximization (OSEM) algorithm with 3 iterations and 21 subsets to a standard clinical voxel size of $2.09 \times 2.09 \times 2.03 \mathrm{~mm}^{3}$ and image size of $344 \times 344 \times 127$. Data from six subjects were excluded from PET reconstruction either due to problems with the PET acquisition $(n=3)$ or due to failure of the Dixon fat/water classification $(n=3)$. Analysis of segmentation and PET results was performed on the remaining 92 subjects.

\subsection{Data Analysis}

The analysis in this study was designed according to previously presented guidelines [5] for evaluating MR-based attenuation correction methods and was conducted using MATLAB software.

2.6.1 Segmentation of Bone and Air-RiDR segmentations of bone and air were derived for each subject using the procedure described in Section 2.2. CT segmentations were derived from CT images by classifying voxels greater than $300 \mathrm{HU}$ as bone and voxels less than $-500 \mathrm{HU}$ as air [19]. The segmentation accuracy of the MR methods (RiDR and vUTE) was analyzed by computing Dice coefficients (Equation 4) for bone and air segmentations with respect to the CT-based method. The numerator of Equation 4 isolates the intersection, or overlap, between MR and CT classifications, while the denominator corresponds to the total number of voxels identified by both modalities. The sample mean and standard deviation (SD) of the Dice coefficients were then computed for the RiDR method.

$$
\text { Dice coefficient }=\frac{2 \cdot(M R \cap C T)}{M R+C T}
$$

2.6.2 R2*-to-CT Regression-The strength of the regressions in Section 2.3 was measured by the coefficient-of-determination $\left(\mathrm{r}^{2}\right)$, and the accuracy of the model to estimate bone CT-HU values was evaluated via a percent-error comparison with acquired CT images. In order to study the improvement provided by the R $2 *$-to-CT conversion, the accuracy of assigning a single CT value to all bone voxels was also evaluated. First, the CT-HU value 
corresponding to the routinely assigned LAC value of $0.151 \mathrm{~cm}^{-1}$ [19] was found through Equation 3 to be $1037 \mathrm{HU}$. Next, a percent-error computation was performed between the CT-HU values of bone voxels and this constant value. The mean percent-error across subjects was computed for the CAR method and the constant method to gauge the improvement provided by the CAR method.

2.6.3 Whole-brain PET Errors-Percent-error maps were computed for PET images

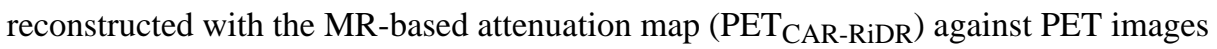
reconstructed with the gold standard $\left(\mathrm{PET}_{\mathrm{CT}}\right)$. From these error maps, the mean absolute percent-error (MAPE) in whole-brain (Equation 5) was computed for each subject, similar to [25], followed by the sample mean and SD. In order to measure the range of errors, the difference between the $95^{\text {th }}$ and $5^{\text {th }}$ percentiles was computed for each subject, followed by the sample mean and SD.

$$
M A P E(\%)=\frac{\sum_{i=1}^{n} 100 \cdot \frac{\left|P E T_{C A R-R i D R}-P E T_{C T}\right| i}{\left(P E T_{C T}\right) i}}{\sum_{i=1}^{n} i}, n=\text { number of brain voxels }
$$

In addition to error maps, voxel-wise correlations between CT-based reconstructions and MR-based reconstructions were also computed. The PET $_{\mathrm{CT}}$ signal intensities of brain voxels

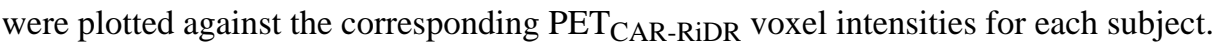
Linear regressions were performed, and the slopes of the resulting "lines-of-best-fit" were used to further characterize the accuracy of the proposed method.

2.6.4 Regional PET Errors-The ICBM 2009c nonlinear symmetric brain atlas (McConnell Brain Imaging Centre, Montreal, Canada), which contains detailed anatomical labeling, was chosen to define several regions-of-interest (ROIs) for regional PET error analysis. This atlas was aligned to subject PET images through a series of nonlinear (ANTS, PICSL, Philadelphia, PA) and linear (FSL Toolbox, FMRIB, Oxford, United Kingdom) registrations using patient T1-MR images. Once aligned, 24 brain regions (Figure 7) were selected and the MAPE calculations were repeated for these ROIs.

In order to visualize the directionality of the errors (under vs. overestimation) in these ROIs, the mean percent-error (MPE) was computed for all ROIs (Equation 6) in each subject, followed by the sample mean across subjects. Box-and-whisker plots were generated for these sample mean data for each ROI, and outliers were defined as subjects with errors larger in magnitude than 1.5 times the interquartile $\left(75^{\text {th }}-25^{\text {th }}\right.$ percentile) range (IQR).

$$
\operatorname{MPE}(\%)=\frac{\sum_{i=1}^{n} 100 \cdot \frac{\left(P E T_{C A R-R i D R}-P E T_{C T}\right) i}{\left(P E T_{C T}\right)} i}{\sum_{i=1}^{n} i}, n=\text { number of brain voxels }
$$


2.6.5 Statistical Analysis-The statistical comparisons between results were analyzed using two-tailed paired Student's t-tests. A result was considered to be statistically significant when the $\mathrm{p}$-value of the $\mathrm{t}$-test was less than 0.05 .

\section{Results}

\subsection{Segmentation of Bone and Air}

Mean Dice coefficients $( \pm \mathrm{SD})$ across subjects for the RiDR method were $0.75( \pm 0.05)$ for bone and $0.60( \pm 0.08)$ for air. Representative results for one subject (Figure 3$)$ show good agreement between the proposed method and CT-based segmentation for both bone and air.

\subsection{Regression}

The mean and SD values across all subjects of the five parameters in the sigmoid model derived for each subject are shown in Table 1. As can be seen from the low SDs exhibited by each of the five parameters, the parameters are highly consistent across subjects.

The sample mean percent-error $( \pm \mathrm{SD})$ in the estimation of CT-HU was $28.2 \%( \pm 3.0)$ for the CAR method and $46.9 \%( \pm 5.8)$ for the method employing constant CT-HU values $\left(\mathrm{p}<10^{-6}\right)$.

\subsection{Attenuation Maps}

Representative slices from the attenuation maps from one subject (Figure 4) provide qualitative confirmation of accurate segmentation of bone and air voxels in the $\mu_{\text {CAR-RiDR }}$ map compared to $\mu_{\mathrm{CT}}$. Visual inspection of the $\mu \mathrm{CAR}-\mathrm{RiDR}_{\text {map }}$ (Figure 4B) provides confirmation of the accurate representation of continuous bone LACs using the CAR conversion method.

\subsection{Whole-brain PET Errors}

The whole-brain MAPE $( \pm \mathrm{SD})$ value across subjects for the $\mathrm{PET}_{\mathrm{CAR}-\mathrm{RiDR}}$ reconstructions relative to the $\mathrm{PET}_{\mathrm{CT}}$ reconstructions was $2.55 \%( \pm 0.86)$. The difference between the $95^{\text {th }}$ and $5^{\text {th }}$ percentile $( \pm \mathrm{SD})$ error values across subjects in whole-brain as illustrated in Figure 5 .

Figure 6 shows a scatter plot of PET $_{\mathrm{CT}}$ vs. PET $_{\mathrm{CAR} \text {-RiDR }}$ for a representative subject. As

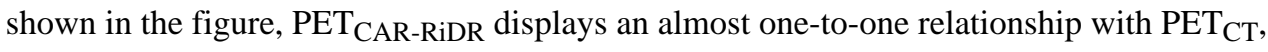
indicating a good correlation between the two methods. The mean slope $( \pm \mathrm{SD})$ across

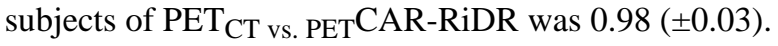

\subsection{Regional PET Errors}

The results from ROI analysis of the mean MAPE values across subjects are in good agreement with the results from the whole-brain analysis presented in Section 3.4. The regional MAPEs (Figure 7, blue circles) in the PET $_{\text {CAR-RiDR }}$ reconstructions ranged from 0.88 to $3.79 \%$ in the 24 ROIs studied, indicating that the CAR-RiDR method is consistently accurate throughout the brain. The regional MPEs (Figure 7, box plot) indicate that the four lobes of the cortex exhibited slightly negative median errors ( 1-2\%), while the cerebellum exhibited slightly larger negative errors ( 2-3\%). Deep gray matter structures such as the 
thalamus and fornix displayed positive median errors compared to the generally negative median errors displayed by regions closer to the brain surface. The number of outliers in each ROI was small relative to the total number of subjects in the study.

\section{Discussion}

Two of the major challenges in MR-based attenuation correction in the head are proper identification of bone and air and accurate estimation of bone LACs. The proposed method CAR-RiDR, developed to address these challenges, consists of two components. The first component RiDR is a method for accurate bone/air segmentation based on intermediate images derived from UTE and Dixon data. The second component CAR is a mechanism for the conversion of bone UTE data in the form of R2* values to CT-HU values, which are subsequently translated to continuous PET LAC values.

Segmentation-based methods for AC rely on accurate segmentation of MR images, the performance of which can be quantitatively measured using Dice coefficients. Based on the mean Dice coefficients reported in Section 3.1, the proposed RiDR segmentation method performs well in identifying both bone and air voxels. Qualitatively, visual overlap between CT-based segmentation and MR-based segmentations of bone and air (Figure 3) can be used to identify degree of misclassification. The RiDR method exhibits a high degree of similarity (yellow) with CT in regions of bone but overestimates the amount of bone (green) in sinus soft tissue. These errors may be due to susceptibility artifacts occurring near airtissue interfaces, which result in abnormally high R2* values and lead to classification errors. The RiDR method also exhibits a high degree of similarity (yellow) with CT in regions of air.

The RiDR segmentation method employs static thresholding of four intermediate images in order to arrive at classifications for bone, air, fat, and soft tissue. These thresholds were empirically selected to minimize misclassifications (Sections 2.2.1-2.2.4), and led to low Dice coefficient standard deviations (Section 3.1) for the RiDR method. Thresholding of MR images is not commonly used due to the sensitivity of voxel intensities to acquisition conditions, thereby making inter-subject comparison of intensities difficult. The RiDR method overcomes this limitation by basing its segmentation in part on normalized intermediate images. For air segmentation, iUTE images were normalized to the $99^{\text {th }}$ percentile value (Section 2.2.1). For bone segmentation, normalization is achieved by virtue of R2* being a physical parameter. While the intermediate images for fat and soft tissue were not normalized, good segmentations were still achieved with static thresholds. The advantage of static thresholds is they remove the need for operator involvement in the segmentation process and automate the RiDR segmentation component of the proposed method.

The CAR method employs a five-parameter sigmoid equation to convert measured patient $\mathrm{R} 2 *$ values into estimated CT-HU values in bone. The parameters for this equation were computed from regression analysis of CT-HU and R2* values from a population of subjects. Validation of this model, which was performed using a leave-one-out strategy, revealed little variation (Section 3.2) across subjects for each of the five parameters. Thus, the conversion 
equation governing the relationship between $\mathrm{R} 2 *$ and $\mathrm{CT}$ values can remain static across patients. These conversion parameters can be computed offline from an existing population of subjects, thereby automating the conversion component of the proposed method. When used in combination with the static thresholds from the RiDR segmentation method, static conversion parameters result in complete automation of the CAR-RiDR method.

The proposed CAR-RiDR method is a hybrid MRAC method that combines the quickness and robustness of segmentation-based methods with the increased accuracy exhibited by atlas-based methods. It differs from existing segmentation-based methods by providing continuous-valued LACs for bone and differs from existing atlas-based methods by avoiding time-consuming variants of pattern recognition methods to estimate these LACs. The R2* to CT-HU conversion can be pre-defined using population data. For a given subject, this conversion can be directly applied to the R2* maps without the need for image registration. As a result, the total computation time is extremely short $(<15 \mathrm{sec})$. This reduction in computation time allows for better integration of attenuation correction into the PET/MRI clinical workflow.

The proposed CAR-RiDR method results in accurate PET reconstructions when evaluated against the gold standard CT-scaled method. Whole-brain (Figures 5 and 6) and regional analysis (Figure 7) of PET reconstruction errors demonstrates that the proposed method performs well across different brain regions. A previous study by Burgos et al. has shown the vendor-provided UTE-AC method, which uses a very similar VB18 WIP-UTE acquisition protocol, results in a whole-brain error of $11.86 \%$ [28]. Burgos et al. employed a slightly different approach to computing whole-brain error than the method presented in this manuscript (MAPE). Recomputing error using their approach yields a whole-brain error of $2.44 \%$ for the CAR-RiDR method. The UTE-AC method was not replicated for in-depth comparison due to the presence of an updated version of this method (VB20), which was not available to us at the time of this study. However, based on the whole-brain error results of Burgos et al., it is clear the proposed CAR-RiDR method produces much more accurate PET reconstructions than the vendor-provided VB18 UTE-AC method.

Direct comparisons between the accuracy of the proposed method and other methods in literature were not performed due to differences in data and in the reference method used for error computations. Errors for segmentation-based methods are typically computed against the "silver standard" [19] CT-segmented method, while atlas-based methods are generally compared to the gold standard. To generate the silver standard attenuation map, a CT image is segmented (as opposed to scaled), and the resulting segmentation is assigned a constant LAC value by tissue class, including bone. The true error (i.e. error when compared to the gold standard) for these methods is therefore unknown.

Recently, a few methods $[21,25]$ combining both atlas- and segmentation-based approaches have been presented. Poynton et al. [21] integrated an atlas-based tissue probability map into a previously presented segmentation-based method [19], improving the accuracy compared to the segmentation-based method alone. However, the analysis in this study was performed against the aforementioned silver standard. Combining T1-MPRAGE and SPM atlas information, Izquierdo-Garcia et al. [25] have reported a MR based attenuation correction 
method that can achieve a good accuracy in PET AC (MAPE $=3.9 \%$ in the whole brain) with a computation time of 30 minutes per attenuation map. Our proposed CAR-RiDR method produces comparable PET errors (MAPE $=2.6 \%$ in the whole brain) with a computation time of less than 15 seconds.

There are a few limitations to our study. First, UTE images (the basis for the R2* maps used both for segmentation and for conversion to CT) suffer from susceptibility artifacts as well as noise issues. These artifacts and noise result in misclassifications of the soft tissue in the sinus regions (Figure 3A). Additionally, images used in this study were acquired mostly from normal subjects at one center using the same PET/MRI and PET/CT scanners. Further evaluation will be needed to determine if differences in the acquisition system or presence of disease states with focal lesions affect the results achieved with the proposed method. The data used in this study were primarily from older subjects (mean age: 66 years \pm 9.8 ). Thus, further validation of the proposed method in more diverse populations is needed. Incorporation of additional population demographics will allow multivariable regression analysis of R2* and CT-HU values, which may reveal demographic-dependent variation of the conversion parameters. Finally, cerebellar regions display the highest ROI errors (median MPEs of -2 to $-3 \%$ ) across patients. Because of their spatial proximity to petrous bone, the cerebellar regions are more subject to bone/air misclassification. This is potentially problematic in clinical applications where PET signal in the cerebellum is used for normalization, resulting in propagation of error to the rest of the brain. Therefore, caution needs to be taken in such normalization.

To our knowledge, the proposed method is the first MR-based attenuation correction method to directly associate the MR relaxation rate $\mathrm{R} 2 *$ with CT-HU in bone, providing continuousvalued attenuation coefficients for bone using only patient information. A previous study [23] examined a joint histogram of R2* maps and co-registered CT images and noted a potential relationship for voxels with a CT value of greater than 100 Hounsfield units (HU), i.e. bone. However, until now, there have been no MR-based attenuation correction methods presented that estimate LAC values for bone based on R2* values. The proposed method has been shown to be highly accurate, producing $<3 \%$ error in whole-brain. Moreover, our method greatly decreases the spatial variations of PET errors as evidenced by the reduction in the difference between the $95^{\text {th }}$ and $5^{\text {th }}$ percentiles of the PET errors (Section 3.4). In contrast to other studies of experimental MRAC methods reported in literature, the accuracy of the CAR-RiDR method has been validated using data from a large number of subjects (n =92). Based on the large dataset used, we can be confident that the results presented in this study are a good measure of the performance of the method.

\section{Conclusions}

In summary, we propose an MR-based attenuation correction method (CAR-RiDR) for use in quantitative PET neurological imaging. The CAR-RiDR method employs UTE and Dixon images and consists of two novel components: 1) accurate segmentation of air and bone using the inverse of the $\mathrm{UTE}^{1}$ image and the R2* image, respectively and 2) estimation of continuous LAC values for bone using a regression between R2* and CT-HU. From our analysis, we conclude the proposed method closely approaches $(<3 \%$ whole-brain error) the 
gold standard CT-scaled method in PET reconstruction accuracy. Additionally, the required UTE images can be acquired quickly $(\sim 1.5 \mathrm{~min})$, and the attenuation maps can be computed rapidly $(<15 \mathrm{sec})$, allowing for ease of incorporation into the PET/MRI clinical work flow.

\section{Acknowledgements}

Funding Support: Siemens Healthcare; The Charles F. and Joanne Knight Alzheimer's Research Initiative; Fred Simmons and Olga Mohan; The Barnes Jewish Hospital Foundation; NIH/NIA P50 AG05681; NIH/NIA P01AG026276; NIH/NIA P01AG003991; NIH/NIA P50 AG05681; NIH 5P30NS048056; NIH 2UL1TR000448; NIH 1R01 NS082561

The authors would also like to acknowledge Avid Radiopharmaceuticals (a wholly owned subsidiary of Eli Lilly) for providing the Florbetapir tracer for the study and for partially funding the scan acquisitions.

\section{References}

1. Catana C, Drzezga A, Heiss W, Rosen BR. PET/MRI for Neurologic Applications. J Nucl Med. 2012; 53:1916-1925. [PubMed: 23143086]

2. Heiss WD. The potential of PET/MR for brain imaging. Eur J Nucl Med Mol Imaging. 2009; (Suppl 1):S105-112. [PubMed: 19104801]

3. Wey HY, Catana C, Hooker JM, Dougherty DD, Knudsen GM, Wang DJ, Chonde DB, Rosen BR, Gollub RL, Kong J. Simultaneous fMRI-PET of the opioidergic pain system in human brain. Neuroimage. 2014; 102P2:275-282. [PubMed: 25107855]

4. Bezrukov I, Mantlik F, Schmidt H, Scholkopf B, Pichler B. MR-based PET attenuation correction for PET/MR Imaging. Semin Nucl Med. 2013; 43:45-49. [PubMed: 23178088]

5. Wagenknecht G, Kaiser H, Mottaghy FM, Herzog H. MRI for attenuation correction in PET: methods and challenges. Magn Reson Mater Phy. 2013; 26:99-113.

6. Keereman V, Mollet P, Berker Y, Volkmar S, Vandenberghe Stefaan. Challenges and current methods for attenuation correction in PET/MR. Magn Reson Mater Phy. 2013; 26:81-98.

7. Bai C, Shao L, Da Silva AJ, Zhao Z. A generalized model for the conversion from CT numbers to linear attenuation coefficients. IEEE Trans Nucl Sci. 2003; 50:1510-1515.

8. Carney JPJ, Townsend DW, Rappoport V, Bendriem B. Method for transforming CT images for attenuation correction in PET/CT imaging. Med Phys. 2006; 33:976-983. [PubMed: 16696474]

9. Kinahan PE, Townsend DW, Beyer T, Sashin D. Attenuation correction for a combined 3D PET/CT scanner. Med Phys. 1998; 25:2046-2053. [PubMed: 9800714]

10. Akbarzadeh A, Ay MR, Ahmadian A, Alam NR, Zaidi H. MRI-guided attenuation correction in whole-body PET/MR: assessment of the effect of bone attenuation. Ann Nucl Med. 2013; 27:152162. [PubMed: 23264064]

11. Andersen FL, Ladefoged CN, Beyer T, Keller SH, Hansen AE, Højgaard L, Kjær A, Law I, Holm S. Combined PET/MR imaging in neurology: MR-based attenuation correction implies a strong spatial bias when ignoring bone. Neuroimage. 2014; 84:206-216. [PubMed: 23994317]

12. Rota Kops E, Herzog H. Alternative methods for attenuation correction for PET images in MRPET scanners. IEEE Nucl Sci Conf R. 2007:4327-4330.

13. Hofmann M, Steinke F, Scheel V, Charpiat G, Farquhar J, Aschoff P, Brady M, Schölkopf B, Pichler BJ. MRI-Bases Attenuation Correction for PET/MRI: A Novel Approach Combining Pattern Recognition and Atlas Registration. J Nucl Med. 2008; 49:1875-1883. [PubMed: 18927326]

14. Schreibmann E, Nye JA, Schuster DM, Martin DR, Votaw J, Fox T. MR-based attenuation correction for hybrid PET-MR brain imaging systems using deformable image registration. Med Phys. 2010; 37:2101-2019. [PubMed: 20527543]

15. Chen Y, Juttukonda M, Su Y, Benzinger T, Rubin BG, Lee YZ, Lin W, Shen D, Lalush D, An H. PASSR: Probabilistic Air Segmentation and Sparse Regression estimated pseudo CT for PET/MR attenuation correction. Radiology. 2014 doi: 10.1148/radiol.14140810. 
16. Martinez-Möller A, Souvatzoglou M, Delso G, Bundschuh R, Chefd'hotel C, Ziegler S, Navab N, Schwaiger M, Nekolla S. Tissue classification as a potential approach for attenuation correction in whole-body PET/MRI: evaluation with PET/CT data. J Nucl Med. 2009; 50:520-526. [PubMed: 19289430]

17. Eiber M, Martinez-Möller A, Souvatzoglou M, Holzapfel K, Pickhard A, Loffelbein D, Santi I, Rummeny E, Ziegler S, Schwaiger M, Nekolla S, Beer A. Value of a Dixon-based MR/PET attenuation correction sequence for the locatlization and evaluation of PET-positive lesions. Eur J Nucl Med Mol Imaging. 2011; 38:1691-1701. [PubMed: 21688050]

18. Keereman V, Fierens Y, Broux T, Deene YD, Lonneux M, Vandenberghe S. MRI-Based Attenuation Correction for PET/MRI Using Ultrashort Echo Time Sequences. J Nucl Med. 2010; 51:812-818. [PubMed: 20439508]

19. Catana C, van der Kouwe A, Benner T, Michel CJ, Hamm M, Fenchel M, Fischl B, Rosen B, Schmand M, Sorensen AG. Toward Implementing an MRI-Based PET Attenuation-Correction Method for Neurologic Studies on the MR-PET Brain Prototype. J Nucl Med. 2010; 51:14311438. [PubMed: 20810759]

20. Berker Y, Franke J, Salomon A, Palmowski M, Donker HCW, Temur Y, Mottaghy FM, Kuhl C, Izquierdo-Garcia D, Fayad ZA, Kiessling F, Schulz V. MRI-Based Attenuation Correction for Hybrid PET/MRI Systems: A 4-Class Tissue Segmentation Technique Using a Combined Ultrashort-Echo-Time/Dixon MRI Sequence. J Nucl Med. 2012; 53:796-804. [PubMed: 22505568]

21. Johansson A, Karlsson M, Nyholm T. CT substitute derived from MRI sequences with ultrashort echo time. Med Phys. 2011; 38:2708-2714. [PubMed: 21776807]

22. Poynton CB, Chen KT, Chonde DB, Izquierdo-Garcia D, Gollub RL, Gerstner ER, Batchelor TT, Catana C. Probabilistic atlas-based segmentation of combined T1-weighted and DUTE MRI for calculation of head attenuation maps in integrated PET/MRI scanners. Am J Nucl Med Mol Imaging. 2014; 4:160-171. [PubMed: 24753982]

23. Delso G, Carl M, Wiesinger F, Sacolick L, Porto M, Hüllner M, Boss A, Veit-Haibach P. Anatomic Evaluation of 3-Dimensional Ultrashort-Echo-Time Bone Maps for PET/MR Attenuation Correction. J Nucl Med. 2014; 55:780-785. [PubMed: 24639457]

24. Horch RA, Nyman JS, Gochberg DF, Dortch RD, Does MD. Characterization of 1H NMR signal in human cortical bone for magnetic resonance imaging. Magn Reson Med. 2010; 64:680-687. [PubMed: 20806375]

25. Izquierdo-Garcia D, Hansen AE, Förster S, Benoit D, Schachoff S, Fürst, Chen KT, Chonde DB, Catana C. An SPM8-Based Approach for Attenuation Correction Combining Segmentation and Nonrigid Template Formation: Application to Simultaneous PET/MR Brain Imaging. J Nucl Med. 2014 doi: 10.2967/jnumed.113.136341.

26. Linstrom, PJ.; Mallard, WG. NIST Chemistry WebBook, NIST Standard Reference Database Number 69, National Institute of Standards and Technology, Gaithersburg MD, 20899, http:// webbook.nist.gov, (retrieved November 26, 2014)

27. Wehrl HF, Sauter AW, Judenhofer MS, Pichler BJ. Combined PET/MR Imaging - Technology and Applications. Technol Cancer Res Treat. 2010; 9:5-20. [PubMed: 20082526]

28. Burgos N, Cardoso MJ, Thielemans K, Modat M, Pedemonte S, Dickson J, Barnes A, Ahmed R, Mahoney CJ, Schott JM, Duncan JS, Atkinson D, Arridge SR, Hutton BF, Ourselin S. Attenuation correction synthesis for hybrid PET-MR scanners: application to brain studies. IEEE Trans Med Imaging. 2014; 33:2332-2341. [PubMed: 25055381]

29. Navalpakkam BK, Braun H, Kuwert T, Quick HH. Magnetic resonance-based attenuation correction for PET/MR hybrid imaging using continuous valued attenuation maps. Invest Radiol. 2013; 48:323-332. [PubMed: 23442772]

30. Gottschalk PG, Dunn JR. The five-parameter logistic: A characterization and comparison with the four-parameter logistic. Anal Biochem. 2005; 343:54-65. [PubMed: 15953581] 


\section{Highlights}

- Good bone/air segmentation can be achieved using UTE and Dixon images.

- A sigmoid model is used to estimate continuous CT-HU values using MR R2* values.

- PET reconstructions with a mean error of $2.6 \%$ in whole-brain are produced.

- Attenuation map computation time is less than 15 seconds per map. 

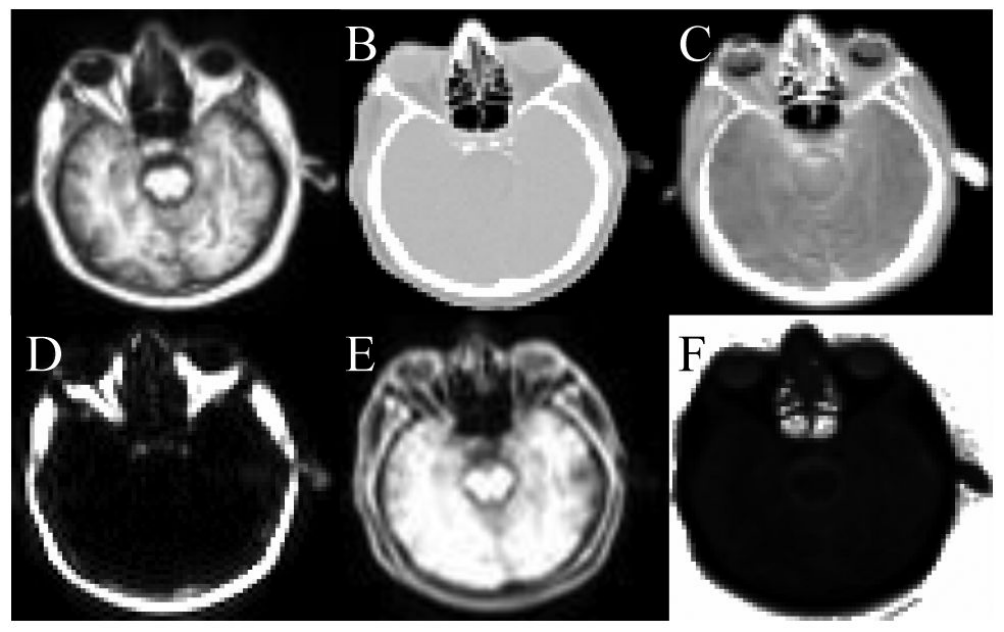

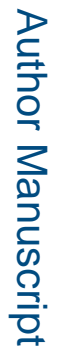

Figure 1.

Sample slices from T1 (A) and CT (B) are shown here along with corresponding slices from R2* (C), Dixon-Fat (D), Dixon-Water (E), and iUTE (F), respectively. 


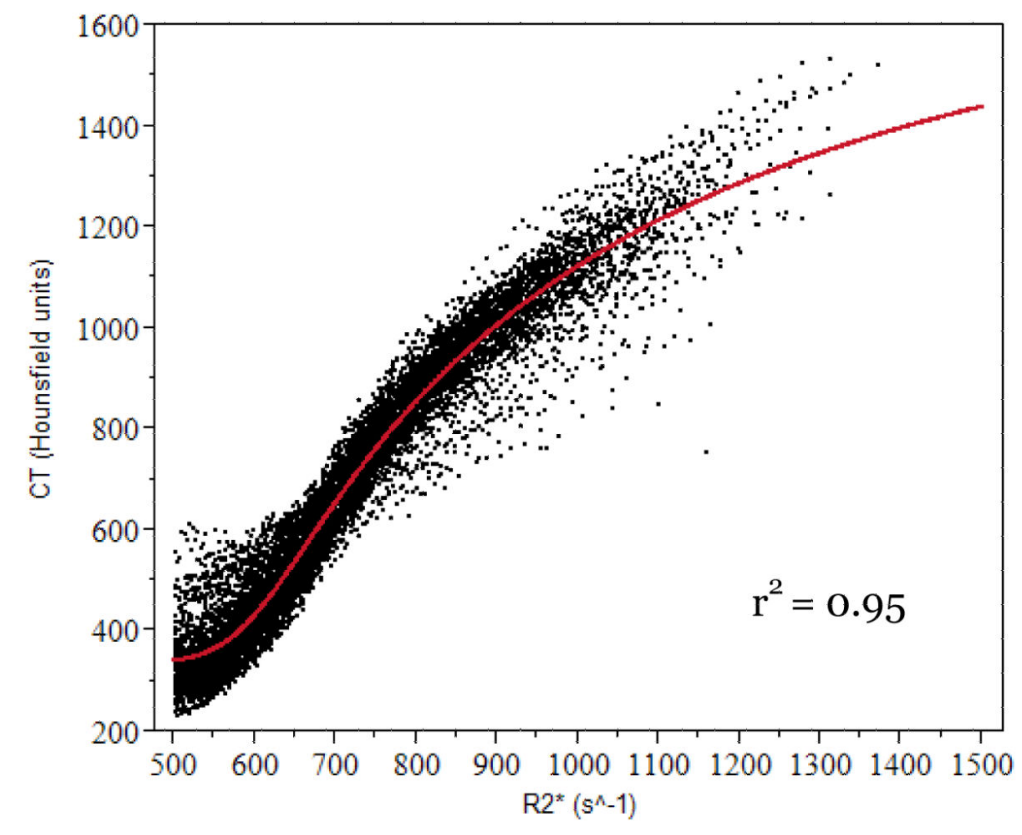

Figure 2.

A mean R2* vs CT-HU scatter plot derived from 97 subjects using a leave-one-out approach suggests a strong sigmoid relationship. Each point represents a mean bin R2* and CT-HU value from one of the 97 subjects. The sigmoid-of-best-fit (red) displays the R2*-to-CT conversion curve used for the left-out subject. 


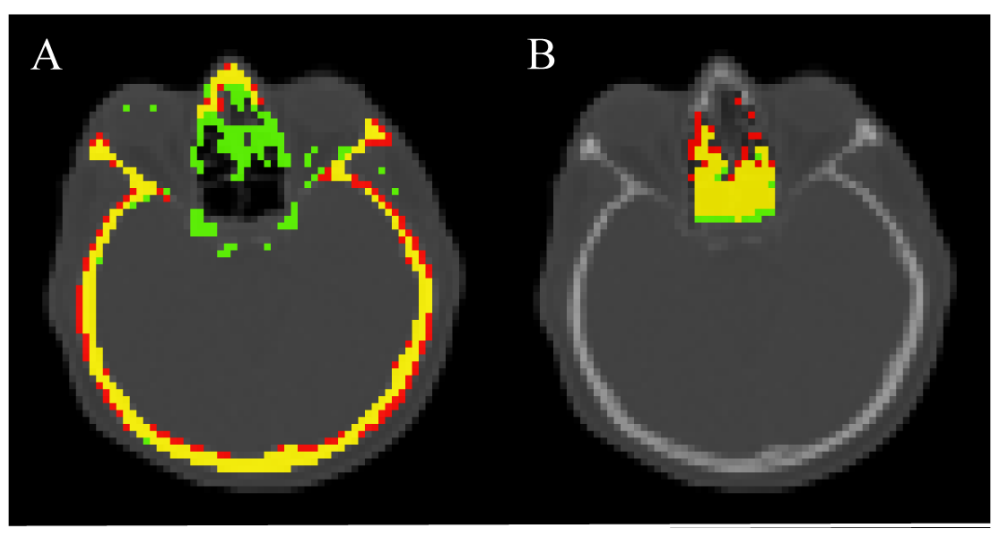

Figure 3.

Representative segmentation results from one subject for the RiDR method were overlaid on CT for bone (A) and air (B). True positives (yellow), false positives (green, overestimation), and false negatives (red, underestimation) are shown. 

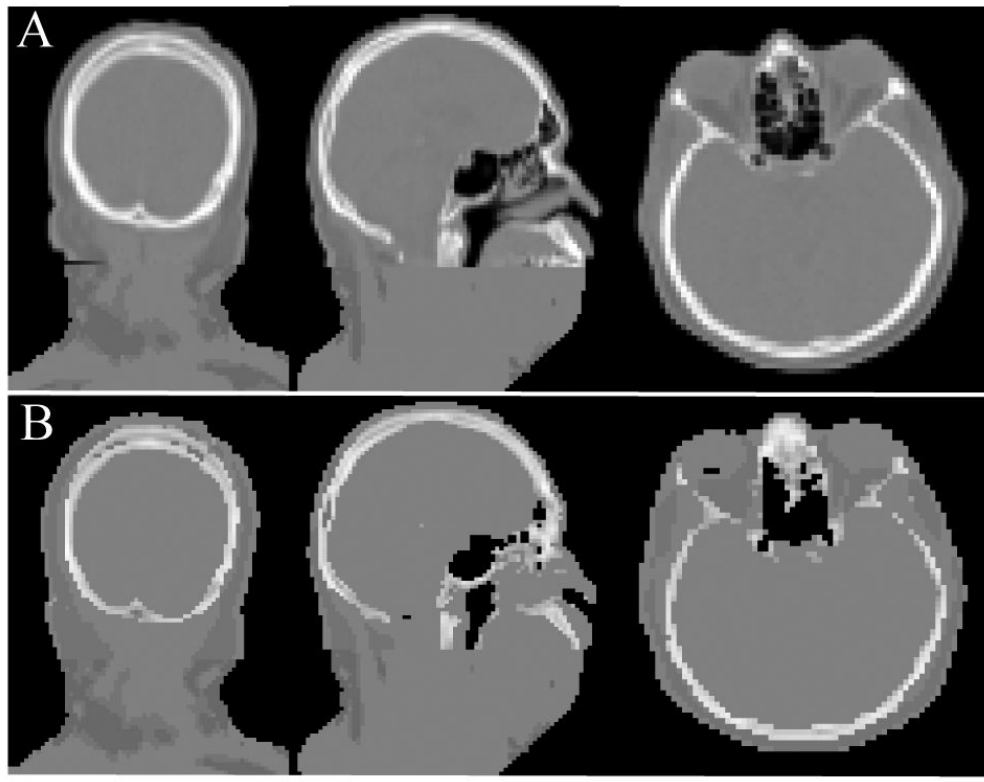

Figure 4.

Sample slices from the $\mu \mathrm{CT}$ (A) and $\mu \mathrm{CAR}-\mathrm{RiDR}$ (B) attenuation maps from one subject are shown in three orientations. 


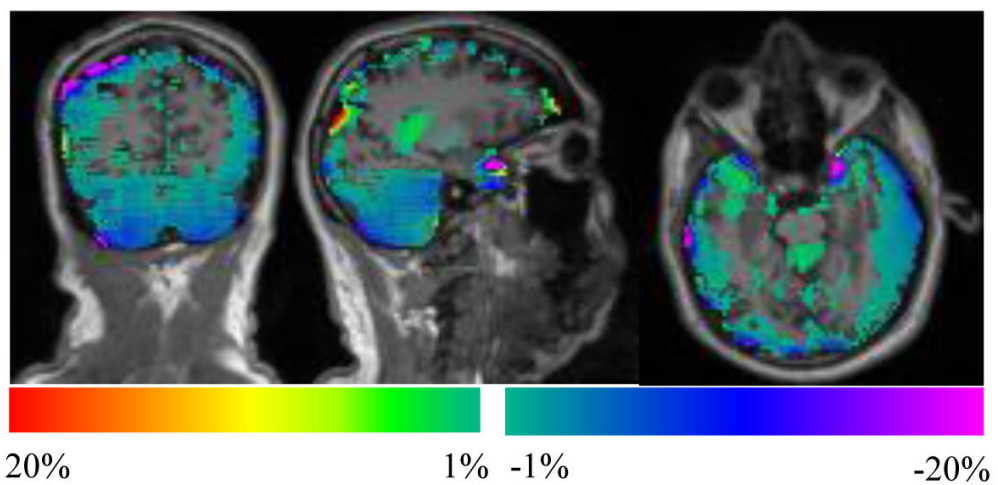

Figure 5.

Representative slices from percent-error maps from one subject show that the CAR-RiDR AC method performs well in most regions of the brain. Errors between $\pm 1 \%$ are suppressed for clarity. 


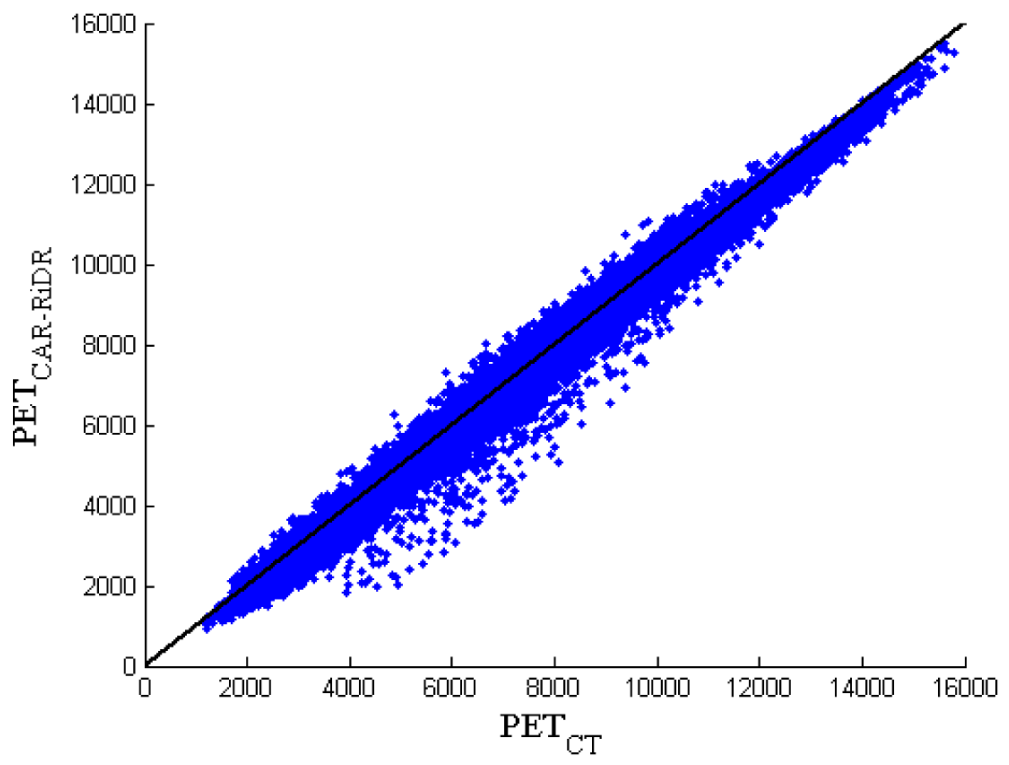

Figure 6.

A voxel-wise scatter plot (blue) of PETCT vs. PETCAR-RiDR brain voxel intensities from a representative subject is shown here. The unity slope (black line) indicates a case with ideal correlation. 


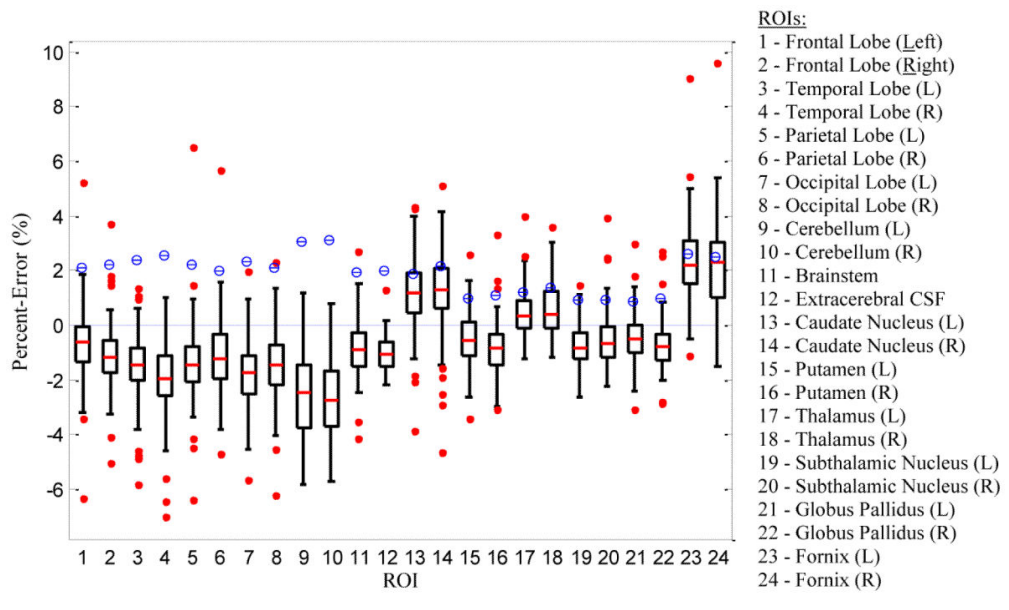

Figure 7.

Results of ROI analysis are shown here using box-and-whisker plots for MPE distributions across all subjects in 24 ROIs. The median (red line), $25^{\text {th }}$ and $75^{\text {th }}$ percentile (box), $1.5 *$ IQR (whiskers), and outliers (red dots) are displayed for each ROI. The mean MAPEs across subjects for each ROI are represented by blue circles. Zero error is indicated by the dashed blue line. 


\section{Table 1}

\begin{tabular}{|c|c|c|c|}
\hline \multirow{3}{*}{ 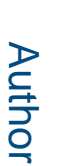 } & \multicolumn{3}{|c|}{ Sigmoid Parameters } \\
\hline & Parameter & Mean & SD \\
\hline & $\mathrm{A}$ & 333.2 & 0.89 \\
\hline 文 & B & 16.9 & 0.15 \\
\hline $\bar{\nabla}$ & $\mathrm{C}$ & 593.5 & 0.62 \\
\hline$\stackrel{-}{\rho}$ & $\mathrm{D}$ & 1851.8 & 18.18 \\
\hline & G & 0.083 & 0.002 \\
\hline
\end{tabular}

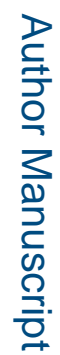

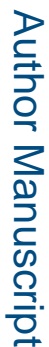

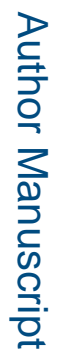

Neuroimage. Author manuscript; available in PMC 2016 May 15. 